\title{
PRIMO CONTRIBUTO PER UNA RETE GRAVIMETRICA FONDAMENTALE IN ITALIA
}

\author{
Carlo Morelli
}

1. Premessa. - Rientra fra i compiti principali dell'Istituto Nazionale di Geofisica l'istituzione in Italia di reti fondamentali nei diversi campi della Geofisica (magnetismo terrestre, elettricita atmosferica, sismologia, gravimetria, ecc.l. In questo programma si inquadra quindi l'istituzione di una serie di stazioni di riferimento per le misure di gravità relativa, dove il valore della gravita fosse noto con la più grande esattezza.

Possibilmente poi cra desiderabile poter predisporre anche una serie di hasi di taratura per i gravimetri destinati alla prospezione geofisica. E noto infatti ( ${ }^{1}$ ) che l'unica difficoltà veramente seria nell'adozione dei gravimetri consiste nella loro taratura (o nel controllo di essal con criteri di sufficiente precisione; e ciò vale in particolare per i gravimetri a grande campo, destinati per i collegamenti a distanze magriori.

Abbiamo già mostrato $(-)$ come le differenze di gravita misurate con $\mathrm{i}$ pendoli non consentano in generale di raggiungere la precisione necessaria, e come il più delle volte l'errore medio pubblicato coi risultati delle misure pendolari abhia un significato puramente apparente; ed abbiamo anche indicato le cause di ciò.

L'uso dei pendoli va quindi scartato anche in questi casi nell'impiego estensivo (tenendo pure conto della laboriosità connessa con tali misurel, e invece limitato alla istituzione di alcuni singoli caposaldi collegati con grande cura e un numero sufficientemente alto di volte, quando si disponga delle apparecchiature necessarie e si adottino i criteri moderni di misura.

Abliamo invece mostrato $(2)$ come, con opportuni criteri d'impiego e convenientemente tarati, $i$ gravimetri geodetici possano ogg $i$ sostituire con vantaggrio i pendoli nella maggior parte dei collegamenti gravimetrici.

L'Istituto Nazionale di Geofisica ha perciò provveduto anzitutto alla taratura, con la massima precisione finora consentita dalle misure pendolari di confronto, dei due gravimetri Worden $n .50$ e n. $52(2)$ 
Il campo di taratura si estende da 980,071 a $981,500 \mathrm{~cm}^{-\mathrm{sec}^{-2}}$ : in tale campo è praticamente compresa tutta l'Italia. Le costanti di scala risultano lineari, e determinate con l'approssimazione di $0,35 \%$.

Contemporaneamente alla taratura dei gravimetri, si è provveduto a collegare con la più grande cura con Potsdam la stazione nazionale di riferimento italiana, col seguente risultato $\left(^{2}\right)$ :

$$
g_{\text {Padova }}=980,658.55+0.37
$$

Tale valore si riferisce alla sala gravimetrica al pianoterra della torre dell'Osservatorio Astronomico ( $h=14,3 \mathrm{~m})$, che $\grave{e}$ m 4,65 più bassa della stazione Lorenzoni alla quale sono stati riferiti (tenendo conto della sola riduzione in aria libera) tutti i precedenti collegamenti pendolari, eseguiti però al pianoterra. Il valore per la stazione Lorenzoni, calcolato in tale maniera, risulta quindi 980,657.1 (*).

2. Criteri d'impiego e scelta delle stazioni. - Abliamo grià mostrato $\left({ }^{2}\right)$ come per $i$ collegamenti a grande distanza con $i$ gravimetri Worden $\dot{e}$ indispensabile il trasporto aereo, sia per consentire una grande rapidití di chiusura dei circuiti (e quindi ridurre al minimo l'incertezza della deriva) come per evitare le condizioni disagiate di trasporto (che possono far variare notevolmente la deriva stessa). I collegamenti eseguiti sono quindi tutti, e solo, quelli che sono consentiti dalle (poche) linee aeree civili oggri esistenti in Italia (v. fig. 1). Essi costituiscono una serie di poligoni chiusi, dagli errori di chiusura dei quali si potrà avere (v. tabella II) un controllo della precisione effettivamente conseguita ed un mezzo per la compensazione dei risultati ( $\$ 3$ ). Mancando una linea aerea, è stato eseguito con automezzo e con i criteri più sotto esposti, il collegamento Padova-Milano, per chiudere il triangolo Padova-Milano-Roma, nonché, data la vicinanza delle stazioni, quello Padova-Trieste. Con automezzo è stata eseruita pure la derivazione Padova-Trento e quella Verona-Trento (v. \$ 4).

Abbiamo anche già visto $\left({ }^{2}\right)$ che con opportuni criteri e ripetizioni il trasporto della gravita fra due posti a piccola distanza (praticamente fino a circa un'ora di macchina) può con sicurezza esser effettuato con l'approssimazione di $0.01 \mathrm{mgal}$, Questi errori sono quindi del tutto trascurabili di fronte a quelli dei collegamenti a grande distanza.

(*) Esso era 980,658.0 - 2.0 dopo la compensazione del Borrass 1909, e 980,656.1 it 0.7 dopo quella dello scrivente (1946). 


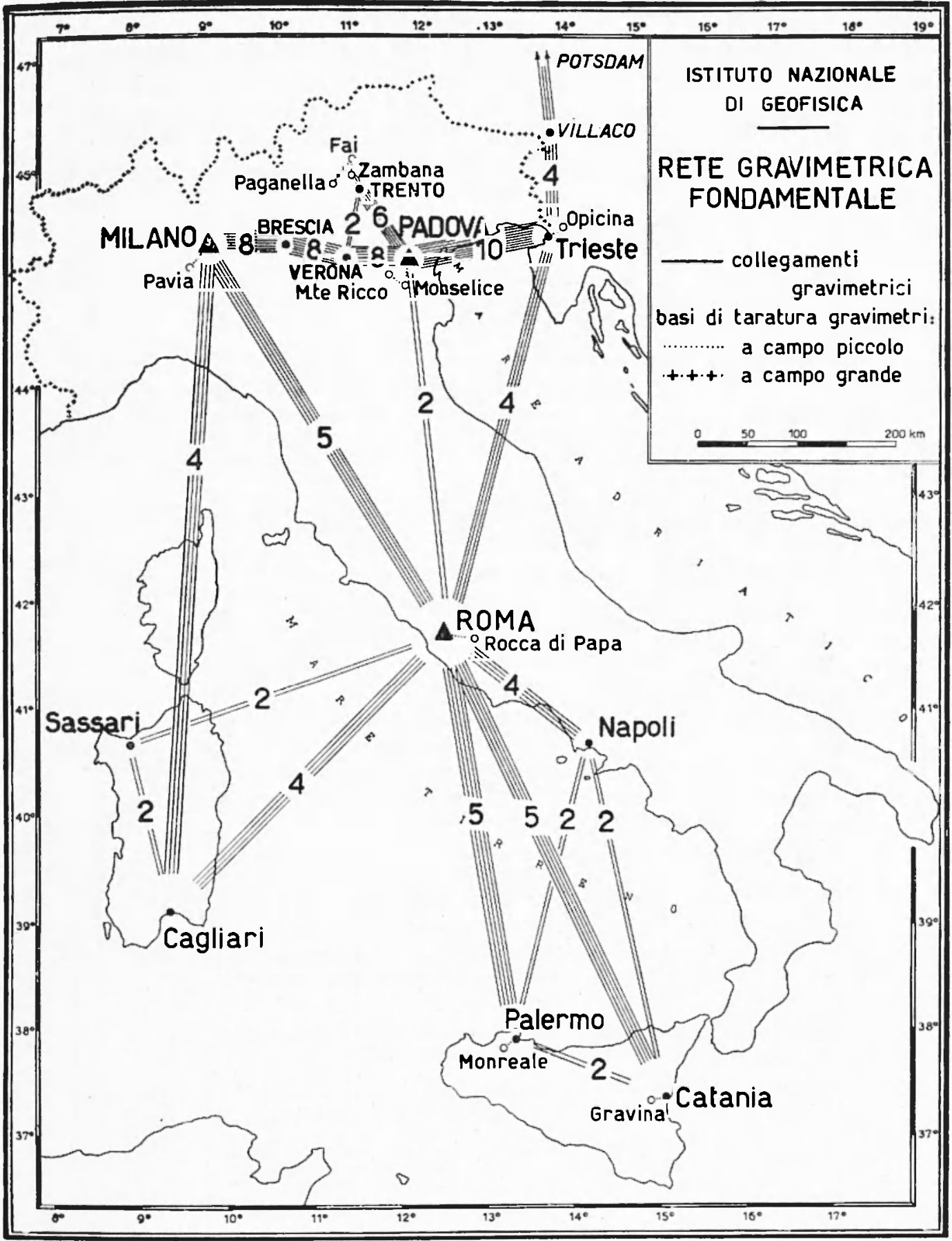

Fig. 1 


\section{Tabella I}

Descrizione delle stazioni gravimetriche principali e sussidiarie.

\begin{tabular}{|c|c|c|}
\hline Stazione & Descrizione & $\begin{array}{l}\text { Numero dellia } \\
\text { monografia }\end{array}$ \\
\hline Breseia & $\begin{array}{l}\text { davanti al marciapiede antistante al portone } 11.11 \\
\text { di piaczal della Vittoria (palazzo dell'INA) }\end{array}$ & (1) \\
\hline CAGLIARI & $\begin{array}{l}\text { ex Istituto Geologico (oral distrutto), bastione adia- } \\
\text { cente all'Lniversiti; davanti all'abitazione del cu- } \\
\text { stode (via Camino nuovo, ll) }\end{array}$ & (2) \\
\hline aereoporto & $\begin{array}{l}\text { edificio sede del C.d.A., angolo a sinistra salendo, } \\
\text { sotto il porticato allal sommiti dellal sealinata }\end{array}$ & (3) \\
\hline CATANIA & $\begin{array}{l}\text { nello scantinato dell'Osservatorio meteoro-sismico, } \\
\text { in piazza Vaccarini n. } 11 \text {, a m. 0,50 davanti al pi- } \\
\text { lastro nell'angolo SE (vano col busto del prof. } \\
\text { Tacchini) }\end{array}$ & (4) \\
\hline aereoporto & $\begin{array}{l}\text { al centro dell'ufficio controllo traffico, nell'edificio } \\
\text { passeggeri a sinistra uscendo dalla pistal }\end{array}$ & (5) \\
\hline Gravina & Municipio, nel mezzo della stanzal angolo $\mathrm{NW}$ & (6) \\
\hline Mascalucia & $\begin{array}{l}\text { itrio della ranonica, a mi } 1.50 \text { dalla parete di fron- } \\
\text { te all'ingresso }\end{array}$ & (7) \\
\hline MILANO & $\begin{array}{l}\text { Istituto di Topograffia e Geodesia del Politecnico, } \\
\text { pilastro gravimetrico in cantinal }\end{array}$ & (8) \\
\hline aereoporto & $\begin{array}{l}\text { Forlanini, porticato sotto la rampa di accesso al- } \\
\text { l'atrio passeggeri, a m l.5 sotto lia finestrellia nel- } \\
\text { l'angolo NE }\end{array}$ & (9) \\
\hline Pavia & $\begin{array}{l}\text { Osservatorio Geofisico, stanzal fotografical a p. } t \text {. } \\
\text { ittigual al locale di affumicatura }\end{array}$ & (10) \\
\hline NAPOLI I & $\begin{array}{l}\text { Osservatorio Astronomico, cantina (ex sala meri- } \\
\text { diani) solto la sala delle conferenze, ai piedi del- } \\
\text { le viti per le mensole bipendolari, a } 40 \mathrm{~cm} \text { dal } \\
\text { muro }\end{array}$ & (11) \\
\hline II & $\begin{array}{l}\text { Università, Stazione Sismical all'Istituto di Fisica } \\
\text { Terrestre (piazza S. Marcellina lo), sul pilastro } \\
\text { ucl terzo locale }\end{array}$ & (12) \\
\hline aereoporto & $\begin{array}{l}\text { a Capodichino: pianoterra edificio LAI, ufficio } \\
\text { informazioni (sotto la finestra) }\end{array}$ & (13) \\
\hline PADOVA I & $\begin{array}{l}\text { Osservatorio Astronomico, stanza gravimetrica nat- } \\
\text { zionale (al pianoterra della torre), angolo SW }\end{array}$ & (14) \\
\hline II & $\begin{array}{l}\text { Universitò, al centro della cantina dell'Istituto di } \\
\text { Geodesia }\end{array}$ & (15) \\
\hline Monselice & marciapiede antistante vecchio Monte di Pieti & (16) \\
\hline
\end{tabular}


(segue tabella I)

\begin{tabular}{|c|c|c|}
\hline Stazione & Descrizione & $\begin{array}{l}\text { Numero della } \\
\text { monografia }\end{array}$ \\
\hline Monte Ricco & $\begin{array}{l}\text { eremo, alla base della scaletta di accesso (lato } \\
\text { Sud) nell'ex rifugio antiaereo }\end{array}$ & (17) \\
\hline PALERMO I & $\begin{array}{l}\text { Palazzo Reale: al centro della parete Sud nella } \\
\text { Sala del Tesoro (praticamente stesso valore di } \mathrm{g} \\
\text { in tutti i punti del ballatoio) }\end{array}$ & (18) \\
\hline II & $\begin{array}{l}\text { Martorana: Istituto di Chimica Industriale alla Fa- } \\
\text { colta d'Ingegneria, nell'aula vicino alla porta in- } \\
\text { terna (locale ricostruito, col pavimento rialzato } \\
\text { di circa } 50 \mathrm{~cm} \text { ) }\end{array}$ & (19) \\
\hline III & $\begin{array}{l}\text { Villa Giulia: all'Ingresso sul Foro Italico, accan- } \\
\text { to al pilastro col es. v. I.G.M. }\end{array}$ & (21) \\
\hline aereoporto & $\begin{array}{l}\text { nell'angolo interno di SE del Servizio Metereo- } \\
\text { logico }\end{array}$ & (20) \\
\hline Monreale & $\begin{array}{l}\text { davanti al portone n. } 2 \text { della piazza Guglielmo II, } \\
\text { fra l'ingresso al Chiostro dei Benedettini e quello } \\
\text { delle Scuole elementari }\end{array}$ & (22) \\
\hline ROMA I & $\begin{array}{l}\text { ex stazione pendolare alla Facolta d'Ingegneria, } \\
\text { S. Pietro in Vincoli; nella cantina gia dell'Istitu- } \\
\text { to di Topografia (ora officina dell'Ist ituto di Scien- } \\
\text { za delle Costruzioni), nell'angolo NNW dell'edif- } \\
\text { cio principale }\end{array}$ & (23) \\
\hline II & $\begin{array}{l}\text { Stazione Sismica all'Istituto Nazionale di Geofisi- } \\
\text { ca in Citti Universitaria, pilastro gravimetrico } \\
\text { (davanti alla porta al } N \text { ) }\end{array}$ & (21) \\
\hline III & $\begin{array}{l}\text { Ufficio Geologico ditalia: a sinistra dell'ingres- } \\
\text { so (entrando), alla Stazione Geofisica }\end{array}$ & (25) \\
\hline \multirow[t]{2}{*}{ aeroporti } & $\begin{array}{l}\text { Ciampino } W \text { : sotto la finestra nellangolo } N \text { della } \\
\text { cosiddetta "sala rosa " (sala arrivi passeggeri) } \\
\text { (stazione Woollard=sala rosa- }(0.15-0.01) \text { mgal) }\end{array}$ & (26) \\
\hline & $\begin{array}{l}\text { Urbe: edificio LAI, stanzetta fra ufficio caposcalo } \\
\text { c bar, ad } 1 \mathrm{~m} \text { dallo spigolo centrale, azimut NNE }\end{array}$ & $(26 a)$ \\
\hline S. Alessio & $\begin{array}{l}\text { Stazione registratrice per l'elettriciti atmosferica } \\
\text { dell'I.N.G, centro stanzetta di NE attigua ai regi- } \\
\text { stratori }\end{array}$ & (27) \\
\hline Frascati & $\begin{array}{l}\text { sotto la finestra } S \text { nel portone (n. 6) del Muni- } \\
\text { cipio (Stazione esterna }=\text { stacione interna }+(0,10 \\
\cdots, 0,01) \text { mgal) }\end{array}$ & (28) \\
\hline \multirow[t]{2}{*}{ Roceal di Papa } & $\begin{array}{l}\text { inferiore: ai piedi della scala che porta alla can- } \\
\text { tina del bar Angeletto }\end{array}$ & (29) \\
\hline & $\begin{array}{l}\text { Osservatorio Geofisico: prima stanzetta (delle pre- } \\
\text { parazionit a sinistra sotto la scala accanto al si- } \\
\text { smografo Wieckert, a m } 1.50 \text { dallo spigolo E }\end{array}$ & (30) \\
\hline
\end{tabular}


(segue tabella I)

\begin{tabular}{|c|c|c|}
\hline Stazione & Descrizione & Numero della \\
\hline SASSARI & $\begin{array}{l}\text { stazione pendolare, aula } \mathrm{C} \text { a pianterreno della Fa- } \\
\text { coltà di Giurisprudenza, in piazza dell'Universitá }\end{array}$ & (31) \\
\hline aeroporto & $\begin{array}{l}\text { Alghero: casa colonica di fronte all'ingresso, cu- } \\
\text { cina, a destra entrando }\end{array}$ & (32) \\
\hline Trento & $\begin{array}{l}\text { a m } 0.60 \text { dal marciapiede, di fronte alla stazione } \\
\text { idrometrica }\end{array}$ & (33) \\
\hline Zambana & $\begin{array}{l}\text { a } 4 \mathrm{~m} \text { dall'ingresso, nella prima galleria (a croce) } \\
\text { adibita a garage, alla stazione inferiore della fu- } \\
\text { nivia }\end{array}$ & (34) \\
\hline Fai & $\begin{array}{l}\text { a } 80 \mathrm{~cm} \text { dalla finestra, sotto il portico a } W \text { della } \\
\text { stazione della funivia }\end{array}$ & (35) \\
\hline Dosso Larici & $\begin{array}{l}\text { al centro della cantina della pensione Dosso La- } \\
\text { rici (stazione terminale funivia Paganella) }\end{array}$ & (36) \\
\hline TRIESTE & $\begin{array}{l}\text { Stazione Sismica dell'Osservatorio Geofisico, sotto } \\
\text { il pilastro del Vicentini verticale } \\
\text { (stazione sismica = ex cantina gravimetrica }-(0,52 \\
+0.01) \text { mgal })\end{array}$ & $(37)$ \\
\hline aereoporto & $\begin{array}{l}\text { Merna (Gorizia): al centro del primo locale a si- } \\
\text { nistra entrando dal cancello principale }\end{array}$ & (38) \\
\hline Opicina & $\begin{array}{l}\text { a m } 1.20 \text { davanti all'ingresso della casa cantoniera } \\
\text { al } \mathrm{km} 12.08 \text { dell'aut ostrada, fra Banne ed Opicinal }\end{array}$ & $(39)$ \\
\hline Verona & Areo di Porta Nuova, al centro verso la città & (40) \\
\hline Vicenza & $\begin{array}{l}\text { a m } 3.50 \text { ad } E \text { spigolo } N E \text { marciapiede antistante } \\
\text { sala pronto soccorso stazione ferroviaria }\end{array}$ & (41) \\
\hline
\end{tabular}

Per le necessità degli orari delle linee aeree, sono state quindi anzitutto istituite stazioni gravimetriche sussidiarie negli aereoporti terminali, si da poter effettuare le misure poco dopo l'arrivo dell'aereo, e poter ripartire con l'aereo successivo. Questi posti sono stati poi collegati con grande cura con la stazione fondamentale vicina.

L'elenco delle stazioni principali (in ordine alfabetico) e di quelle sussidiarie ad esse collegate risulta dalla tabella I. Le monografie corrispondenti sono riportate nelle tre tavole alla fine del testo. II numero $n$ dei collegamenti aerei (contando per uno solo un collegamento completo, in andata e ritorno) risulta dalla tabella II, dove sono riportate pure le differenze di gravità osservate ed il loro errore medio. 
primo contributo Per uNa Rete inavimetrica fondamentale in italia 103

Il collegamento Padova-Milano, effettuato in automezzo, è stato suddiviso per una maggior precisione (conseguente alla rapidità di chiusura dei singoli percorsi) nelle quattro tratte indicate dalla tabella III.

Le differenze di gravità osservate fra le stazioni sussidiarie risultano dalla tabella IV. Lo schema dei colleramenti fra le stazioni sus-

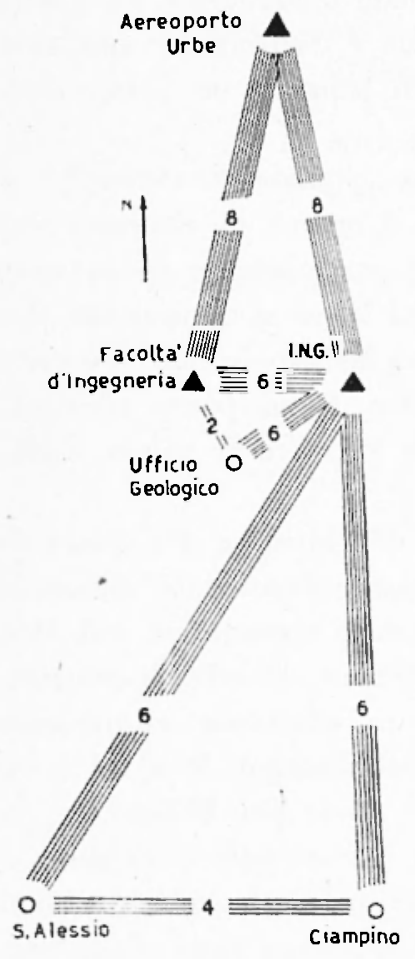

Fig. 2: Collegamenti fra le stazioni sussidiarie in Roma.

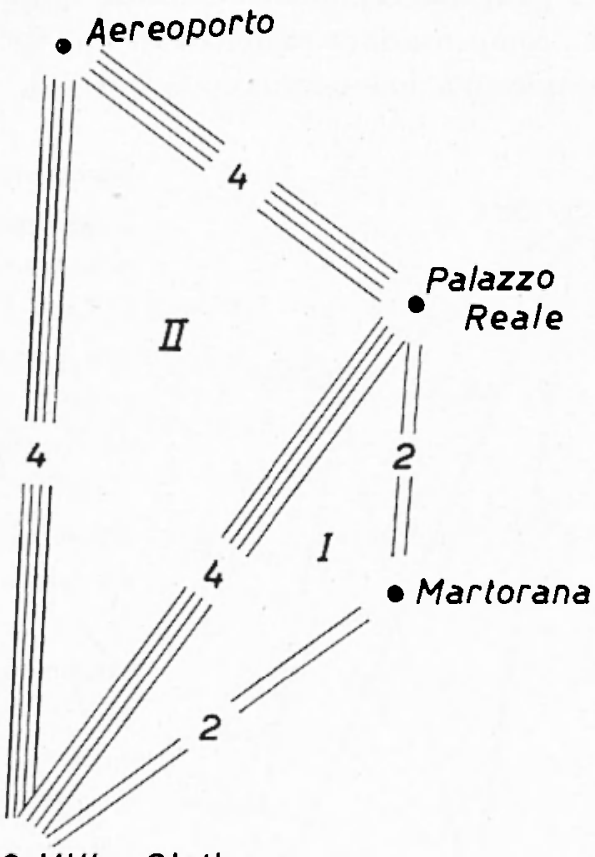

- Villa Giulia

Fig. 3: Collegamenti fra le stazioni sus sidiarie in Palermo.

sidiarie in Roma risulta dalla fig. 2, quello fra le stazioni sussidiarie in Palermo dalla fig. 3 .

3. Precisione. Compensazione dei risultati. - Si vede dalla tabella II che gli errori medi dei collegamenti non sono in nessun caso superiori a $-0.2 \mathrm{mgal}$ : risultato questo superiore ad ogni aspettativa, e che dimostra sia la bontà dei criteri seguiti durante l'esecuzione delle misure, come il buon accordo fra le costanti di scala dei due gravimetri.

L'alto grado di precisione conseguita risulta anche dagli errori 
TABella II - Differenze di gravità (in mgal) osservate fra le stazioni principali. Valori compensati.

A. Italia Centro-Setr. o Sardegna

\begin{tabular}{|c|c|c|c|c|}
\hline \multirow{2}{*}{ Stazione } & \multirow{2}{*}{$\mathbf{n}$} & \multicolumn{2}{|c|}{$\Delta \mathrm{g}(\mathrm{mgal})$} & \multirow{2}{*}{$\mathrm{g}$} \\
\hline & & misurate & compensate & \\
\hline $\begin{array}{l}\text { Padova I } \\
\text { Trieste } \\
\text { Roma I } \\
\text { Padova I }\end{array}$ & $\begin{array}{r}10 \\
4 \\
2\end{array}$ & $\begin{array}{l}\text { I triangolo } \\
+\quad 6.57+0.04 \\
+303.59 \quad .12 \\
+297.01 \quad .12\end{array}$ & $\begin{array}{r}6.57 \\
-303.54 \\
+296.96\end{array}$ & $\begin{array}{r}980,658.55 \\
, 665.12 \\
, 361.58 \\
, 658.54\end{array}$ \\
\hline Errore di chiusura: & & -0.01 & -0.01 & \\
\hline $\begin{array}{l}\text { Padova I } \\
\text { Milano } \\
\text { Roma I } \\
\text { Padova I }\end{array}$ & $\begin{array}{l}8\left(^{\circ}\right) \\
5 \\
2\end{array}$ & $\begin{array}{l}\text { II triangolo } \\
-94.05 \div 0.02 \\
-202.84 \quad .03 \\
+297.01 \quad .12\end{array}$ & $\begin{array}{r}94.12 \\
-202.84 \\
+296.96\end{array}$ & $\begin{array}{r}980,658.55 \\
, 564.43 \\
, 361.59 \\
, 658.55\end{array}$ \\
\hline Errore di chiusura: & & +0.12 & 0.00 & \\
\hline \multicolumn{5}{|c|}{ Valore finale per Roma I : $980,361.59$} \\
\hline $\begin{array}{l}\text { Roma I } \\
\text { Cagliari } \\
\text { Milano } \\
\text { Roma I }\end{array}$ & $\begin{array}{l}4 \\
4 \\
5\end{array}$ & 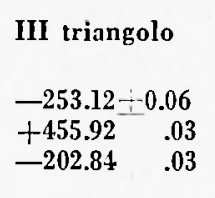 & $\begin{array}{r}-253.07 \\
+455.96 \\
-202.89\end{array}$ & $\begin{array}{r}980,361.59 \\
, 108.52 \\
, 564.48 \\
, 361.59\end{array}$ \\
\hline Errore di chiusura: & & -0.04 & 0.00 & \\
\hline \multicolumn{5}{|c|}{ Valore finale per Milano : $980,56 t .46$} \\
\hline $\begin{array}{l}\text { Roma I } \\
\text { Cagliari } \\
\text { Sassari } \\
\text { Roma I }\end{array}$ & $\begin{array}{l}4 \\
2 \\
2\end{array}$ & $\begin{array}{l}\text { IV triangolo } \\
-253.12+0.06 \\
+111.24 \quad .16 \\
+141.82 \quad .17\end{array}$ & $\begin{array}{r}-253.07 \\
+111.24 \\
+141.82\end{array}$ & $\begin{array}{r}980,361.59 \\
, 108.52 \\
, 219.76 \\
, 361.58\end{array}$ \\
\hline Errore di chiusura: & & -0.06 & -0.01 & \\
\hline
\end{tabular}

(") per via di terra. 
PRIMO CONTRIBUto PER UNA aETE ERAVIMETRICA FONDAMENTALE IN ITALIA 107

B. Italia Merid. e Sicilia

\begin{tabular}{|c|c|c|c|c|}
\hline \multirow{2}{*}{ Stazione } & \multirow{2}{*}{$\mathbf{n}$} & \multicolumn{2}{|c|}{$\Delta_{\mathrm{g}}(\mathbf{m g a l})$} & \multirow{2}{*}{ g } \\
\hline & & misurate & compensate & \\
\hline $\begin{array}{l}\text { Roma I } \\
\text { Palermo } \\
\text { Catania } \\
\text { Roma I }\end{array}$ & $\begin{array}{l}5 \\
2 \\
4\end{array}$ & 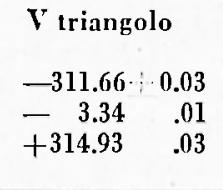 & $\begin{array}{r}-311.64 \\
-\quad 3.30 \\
+314.94\end{array}$ & $\begin{array}{r}980,361.59 \\
, 049.95 \\
, 046.65 \\
, 361.59\end{array}$ \\
\hline Errore di chiusura: & & -0.07 & 0.00 & \\
\hline $\begin{array}{l}\text { Roma I } \\
\text { Napoli I } \\
\text { Catania } \\
\text { Roma I }\end{array}$ & $\begin{array}{l}4 \\
2 \\
4\end{array}$ & 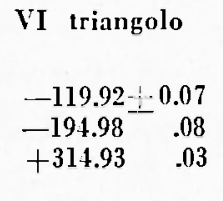 & $\begin{array}{l}-119.94 \\
-195.00 \\
+314.94\end{array}$ & $\begin{array}{r}980,361.59 \\
, 241.65 \\
.046 .65 \\
, 361.59\end{array}$ \\
\hline Errore di chiusura: & & +0.03 & 0.00 & \\
\hline $\begin{array}{l}\text { Roma I } \\
\text { Napoli I } \\
\text { Palermo } \\
\text { Roma I }\end{array}$ & $\begin{array}{l}4 \\
2 \\
5\end{array}$ & $\begin{array}{l}\text { VII triangolo } \\
\begin{array}{rr} \\
-119.92 & -0.07 \\
-191.69 & .04 \\
+311.66 & .03\end{array}\end{array}$ & $\begin{array}{r}-119.94 \\
-191.70 \\
+311.64\end{array}$ & $\begin{array}{r}980,361.59 \\
, 241.65 \\
, 049.95 \\
, 361.59\end{array}$ \\
\hline Errore di chiusura: & & +0.05 & 0.00 & \\
\hline $\begin{array}{l}\text { Napoli I } \\
\text { Palermo } \\
\text { Catania } \\
\text { Napoli I }\end{array}$ & $\begin{array}{l}2 \\
2 \\
2\end{array}$ & $\begin{array}{l}\text { VIII triangolo } \\
\begin{array}{rr} & \\
-191.69 & 0.04 \\
-\quad 3.34 & .01 \\
+194.98 & .08\end{array}\end{array}$ & $\begin{array}{r}-191.70 \\
-\quad 3.30 \\
+195.00\end{array}$ & $\begin{array}{r}980.241 .65 \\
\$ 049.95 \\
, 046.65 \\
, 241.65\end{array}$ \\
\hline Errore di chiusura: & & -0.05 & 0.00 & \\
\hline $\begin{array}{l}\text { Roma I } \\
\text { Palermo } \\
\text { Catania } \\
\text { Napoli I } \\
\text { Roma I }\end{array}$ & $\begin{array}{l}5 \\
2 \\
2 \\
4\end{array}$ & 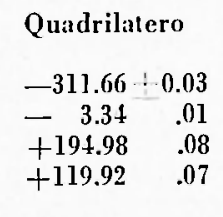 & $\begin{array}{r}-311.64 \\
-\quad 3.30 \\
+195.00 \\
+119.94\end{array}$ & $\begin{array}{r}980,361.59 \\
, 049.95 \\
, 046.65 \\
, 241.65 \\
, 361.59\end{array}$ \\
\hline Errore di chiusura: & & -0.10 & 0.00 & \\
\hline
\end{tabular}


Disponendo le equazioni di condizione nello specchio seguente:

\begin{tabular}{|c|c|c|c|c|c|c|c|c|}
\hline Corr. & Equaz. & $x_{1}$ & $x_{9}$ & $x_{3}$ & $x_{i}$ & $x_{n}$ & $x_{i i}$ & $\mathbf{v}$ \\
\hline $\begin{array}{l}\mathbf{K}_{1} \\
\mathbf{K}_{2} \\
\mathbf{K}_{3} \\
\mathbf{K}_{2} \\
\mathbf{K}_{n}\end{array}$ & $\begin{array}{l}\text { A } \\
\text { B } \\
\text { C } \\
\text { I) } \\
\text { I: }\end{array}$ & $\begin{array}{l}-1 \\
+1 \\
-1\end{array}$ & $\begin{array}{l}+1 \\
+1 \\
+1\end{array}$ & $\begin{array}{l}+1 \\
\div 1\end{array}$ & $\begin{array}{l}-1 \\
-1 \\
+1\end{array}$ & $\begin{array}{l}-1 \\
+1 \\
+1\end{array}$ & $\begin{array}{l}-1 \\
-1\end{array}$ & $\begin{array}{r}-0.07 \\
+0.03 \\
+0.05 \\
-0.05 \\
-0.10\end{array}$ \\
\hline . & $p$ & 5 & 2 & 4 & 4 & 2 & 2 & \\
\hline
\end{tabular}

si ricavano immediatamente le equazioni correlate, che sono:

$$
\begin{aligned}
& x_{1}=-\frac{1}{5}\left(-K_{1}+K_{3}-K_{5}\right), \\
& x_{2}=-\frac{1}{\frac{2}{2}}\left(K_{1}+K_{4}+K_{5}\right), \\
& x_{3}=-\frac{1}{4}\left(K_{1}+K_{2}\right),
\end{aligned}
$$

\begin{tabular}{|c|c|c|c|c|}
\hline \multirow[b]{2}{*}{ Stazione } & \multirow[b]{2}{*}{$n$} & \multicolumn{2}{|c|}{$\Delta g\left(\mathbf{m g}_{11}\right)$} & \multirow[b]{2}{*}{ gPotsilam } \\
\hline & & osservato & compensato & \\
\hline $\begin{array}{l}\text { Padova I } \\
\text { Vicenza } \\
\text { Verona } \\
\text { Brescia } \\
\text { Milano }\end{array}$ & $\begin{array}{l}8 \\
8 \\
8 \\
8\end{array}$ & $\begin{array}{lr}+23.06 \div 0.02 \\
-16.82 & .01 \\
-43.51 & .01 \\
-56.78 & .02\end{array}$ & $\begin{array}{l}+23.05 \\
-16.83 \\
-43.52 \\
-56.79\end{array}$ & $\begin{array}{r}980.658 .55 \\
.681 .60 \\
, 664.77 \\
, 621.25 \\
.564 .46\end{array}$ \\
\hline \multicolumn{5}{|c|}{ Derivazioni } \\
\hline $\begin{array}{l}\text { Padova I } \\
\text { Trento }\end{array}$ & 6 & $-19.73-0.03$ & & $\begin{array}{r}980.658 .5 \\
.608 .82\end{array}$ \\
\hline $\begin{array}{l}\text { Verona } \\
\text { Trento }\end{array}$ & 2 & $-55.90 \div 0.01$ & & $\begin{array}{r}980,664.77 \\
, 608.87\end{array}$ \\
\hline
\end{tabular}

Tabella III - Collegamenti con automezzo. 
primo contributo per uNa Rete travimetrica fondamentale in italia 109

$$
\begin{aligned}
& x_{4}=-\frac{1}{4}\left(-K_{2}-K_{3}+K_{5}\right), \\
& x_{5}=-\frac{1}{2}\left(-K_{2}+K_{4}+K_{5}\right), \\
& x_{6}=-\frac{1}{2}\left(-K_{3}-K_{4}\right),
\end{aligned}
$$

Introducendo nelle equazioni di condizione questi valori di $x$, si ricava il seguente sistema normale di 5 equazioni nelle 5 incognite $K_{1}(i=1,2, \ldots 5)$ :

$$
\begin{aligned}
& 0,95 \cdot K_{1}+0,25 \cdot K_{2}-0 \cdot 2 \cdot K_{3}+0,5 \cdot K_{4}+0,7 \cdot K_{5}+0,07=0 \\
& 1,0 . K_{2}+0,25 \cdot K_{3}-0,5 \cdot K_{4}-0,75 \cdot K_{5}-0,03=0 \\
& 0,95 \cdot K_{3}+0,5 \cdot K_{4}-0,45 \cdot K_{5}-0,05=0 \\
& 1,5 . K_{1}+1,0 K_{5}+0,05=0 \\
& 1,45 . K_{5}+0,10=0
\end{aligned}
$$

dove i coefficienti della diagonale principale sono la somma dei reciproci dei pesi per i lati di ognuno dei triangoli e per il poligono considerati.

Risolvendolo col metodo di Gauss si ricava il seguente sistema risolvente:

$$
\begin{aligned}
& 0,95 . K_{1}+0,25 . K_{2}-0,2 \quad . K_{3}+0,5 \quad . K_{4}+0,7 \quad . K_{5}+0,07=0 \\
& 0,934 \cdot K_{2}+0,303 \cdot K_{3}-0,632 \cdot K_{1}-0,934 \cdot K_{5}-0,048=0 \\
& 0,810 . K_{3}+0,810 . K_{i}+0 \quad . K_{5}+0=0 \\
& 0 . K_{4}+0 \quad . K_{5}+0=0 \\
& 0 \quad . K_{5}-0,020=0
\end{aligned}
$$

dal quale si ottengono $\mathrm{i}$ seguenti valori per i correlativi $K_{\mathrm{i}}$ :

$$
\begin{aligned}
& K_{1}=-0,080 ; \quad K_{4}=0,000 \\
& K_{2}=+0,044 ; K_{5}=0,000 \\
& K_{3}=+0,024 ;
\end{aligned}
$$

Sostituendo questi valori nel sistema normale, e indicando con $\boldsymbol{V}$ gli errori di chiusura delle sue cinque equazioni, risulta a comprova:

$$
[V V]=0,0000
$$




\section{Tabella IV}

Differenze di gravità osservate con le stazioni sussidiarie.

\begin{tabular}{|c|c|c|c|c|}
\hline Stazione principale & Stazioni sussidiarie & $\mathbf{n}$ & $\Delta \mathrm{g}(\mathrm{mgal})$ & I Potndan \\
\hline Cagliari Univ. & aereoporto & + & $+4.80 \div 0.01$ & $\begin{array}{cc}980,108.52 & 0.39 \\
, 111.32 & 0.39\end{array}$ \\
\hline Catania Oss. & aereoporto & 4 & $+12.27-1-0.01$ & $\begin{array}{r}980.046,65-1.38 \\
, 058.92 \\
.38\end{array}$ \\
\hline Milano Polit. & aereoporto & 12 & $-0.67 \div 0.01$ & $\begin{array}{c}980,56+, 46 \cdot 0.37 \\
, 563.79 \quad .37\end{array}$ \\
\hline Napoli Oss. & $\begin{array}{l}\text { aereoporto } \\
\text { Università }\end{array}$ & $\begin{array}{l}4 \\
6\end{array}$ & $\begin{array}{l}+14.00-0.04 \\
+17.88 \quad .03\end{array}$ & $\begin{array}{rr}980,2+1.65 & -0.39 \\
, 255.65 & .39 \\
, 273.53 & .39\end{array}$ \\
\hline Padova Oss. & $\begin{array}{l}\text { Università } \\
\text { Monselice }\end{array}$ & $\begin{array}{c}12 \\
0\end{array}$ & $\begin{array}{rr}-1.73 & 0.01 \\
+20.65 & .01\end{array}$ & $\begin{array}{rr}980.658 .55 & 0.37 \\
, 656.82 & .37 \\
, 677.47 & .37\end{array}$ \\
\hline \multirow[t]{2}{*}{ Palermo PaI. Reale } & $\begin{array}{l}\text { aereoporto } \\
\text { Villa Giulia } \\
\text { Palazzo Reale }\end{array}$ & $\begin{array}{l}4 \\
4 \\
4\end{array}$ & $\begin{array}{rr}-2.24-0.01 \\
+9.69 & .01 \\
-7.46 & .01\end{array}$ & $\begin{array}{rr}980,049.95 & -0,38 \\
, 047.71 & .38 \\
, 057.40 & .38 \\
, 049.94 & .38\end{array}$ \\
\hline & Errore di chiusura: & & -0.01 & \\
\hline \multirow[t]{2}{*}{ Palermo Pal. Reale } & $\begin{array}{l}\text { Martorana } \\
\text { Villa Giulia } \\
\text { Palazzo Reale }\end{array}$ & $\begin{array}{l}2 \\
2 \\
t\end{array}$ & $\begin{array}{rr}+6.11 & 0.0 .4 \\
+ & 1.39 \\
-7.46 & .01 \\
& .01\end{array}$ & $\begin{array}{cc}980,049.95+0.38 \\
, 056.06 & .39 \\
, 057.45 & .39 \\
, 019.99 & .39\end{array}$ \\
\hline & Errore di chiusura: & & +0.04 & \\
\hline \multirow[t]{2}{*}{ Roma Fac. Ing. } & $\begin{array}{l}\text { I. N. G. } \\
\text { aerop. Urbe } \\
\text { Fac. Ing. }\end{array}$ & $\begin{array}{l}6 \\
8 \\
8\end{array}$ & $\begin{array}{rr}+1.98 & .04 \\
+10.39 & .03 \\
-12.36 & .02\end{array}$ & $\begin{array}{cr}980.361 .59+0.38 \\
, 363.57 & .39 \\
, 373.96 & .39 \\
, 361.60 & .39\end{array}$ \\
\hline & Errore di chiusura: & & +0.01 & \\
\hline
\end{tabular}


PRIMO CONTRIBUTO EF UNA RETE [RAVIMETRICA FONDAMENTALE IN ITALIA 111

\begin{tabular}{|c|c|c|c|c|}
\hline & & & \multicolumn{2}{|c|}{ (segue tabella IV) } \\
\hline Stazione principale & Stazioni sussidiarie & n & $\Delta_{\mathrm{g}}(\mathrm{mgal})$ & gPotsdam \\
\hline & $\begin{array}{l}\text { I. N. G. } \\
\text { S. Alessio } \\
\text { Ciampino } \\
\text { I. N. G. }\end{array}$ & $\begin{array}{l}6 \\
4 \\
6\end{array}$ & $\begin{array}{rr}-5.56 & 0.02 \\
-11.29 & .01 \\
+16.87 & .06\end{array}$ & $\begin{array}{rr}980,363.57 & 0,39 \\
, 358.01 & .39 \\
, 346.72 & .39 \\
, 363.59 & .39\end{array}$ \\
\hline & Errore di chiusura: & & +0.02 & \\
\hline & $\begin{array}{l}\text { I. N. G. } \\
\text { Uff. Geologico } \\
\text { Fac. Ing. } \\
\text { I. N. G. }\end{array}$ & $\begin{array}{l}6 \\
2 \\
6\end{array}$ & 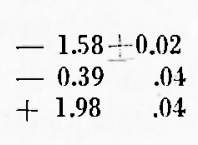 & $\begin{array}{rr}980,363.57 & -0,39 \\
, 361.99 & .39 \\
, 361.60 & .39 \\
, 363.58 & .39\end{array}$ \\
\hline & Errore di chiusura: & & +0.01 & \\
\hline Sassari Univ. & aereoporto & 2 & $+58.34 \div-0.15$ & $\begin{array}{r}980.219 .76-0.42 \\
, 278.10 \quad .45\end{array}$ \\
\hline Trento & Zambana & 4 & $+43.70 \div 0.01$ & $\begin{array}{r}980,608.84-0.37 \\
, 652.54 \quad .37\end{array}$ \\
\hline Trieste Oss. Geof. & aereoporto & 9 & $11.19 \div 0.01$ & $\begin{array}{rr}980,665.12 & 0.36 \\
, 653.93 & .36\end{array}$ \\
\hline
\end{tabular}

Sostituendo invece $\mathrm{i}$ valori $K_{\mathbf{i}}$ sopra ottenuti nelle equazioni correlate, si ricavano $i$ seguenti valori per le correzioni $x_{\mathrm{i}}$ :

$$
\begin{aligned}
& x_{1}=-0,02 \mathrm{mgal} ; x_{t}=+0,02 \mathrm{mgal} ; \\
& x_{2}=+0,04 \quad \text { " ; } x_{5}=+0,02 \% \text {; } \\
& x_{3}=+0,01 \text { » } ; x_{6}=+0,01 \text { ». }
\end{aligned}
$$

Sostituendo questi valori nelle equazioni di condizione, la somma dei quadrati dei residui risulta:

$$
\left[V^{\prime} V^{\prime}\right]=0,0000 \text {. }
$$

Quadrando e sommando i valori ora ottenuti per le correzioni $x_{i}$, si ricava inoltre:

$$
[p x x]=+0,0080 \text {, }
$$

mentre la stessa sommatoria calcolata per controllo mediante la

$$
[p x x]=-\sum_{\mathrm{i}=1}^{5} K_{\mathrm{i}} v_{\mathrm{i}}
$$


fornisce il valore

$$
[p x x]=+0.0081 \text {, }
$$

in ottimo accordo col precedente.

Indicando con $q$ il numero delle equazioni di condizione (cioè, il numero dei poligoni chiusi), l'errore medio $m_{0}$ dell'unità di peso, cioè di un collegamento, diventa:

$$
m_{0}= \pm \sqrt{\frac{[\overline{p x x}]}{q}}= \pm 0.04 \text { mgal . }
$$

Questo risultato è oltremodo soddisfacente, se si considera le grandi dimensioni del quadrilatero completo; e conferma a posteriori la bontà dei criteri di impostazione e di esecuzione della presente ricerca.

Tenendo conto delle correzioni $x_{\mathrm{i}}$ sopra ricavate, le differenze di gravita compensate vengono ad assumere $i$ valori indicati nella penultima colonna della tabella II, e grli errori di chiusura dei triangoli e del quadrilatero diventano tutti nulli: la compensazione eseruita è quindi sufficiente e soddisfacente.

Partendo allora dal valore di $g$ a Roma, i valori della gravità nelle stazioni di riferimento diventano quelli dell'ultima colonna della tabella II. Essi sono riportati nella tabella V con il loro errore medio riferito a Potsdam.

\begin{tabular}{|c|c|}
\hline Stazione & $\mathrm{g}$ \\
\hline $\begin{array}{l}\text { Cagliari } \\
\text { Catania } \\
\text { MILANO } \\
\text { Napoli } \\
\text { PADOVA } \\
\text { Palermo } \\
\text { ROMA } \\
\text { Sassari } \\
\text { Trieste }\end{array}$ & $\begin{array}{cc}980,108.52-1 & 0.39 \\
, 046.65 & .38 \\
, 564.46 & .37 \\
, 241.65 & .39 \\
\mathbf{6} 658.55 & .37 \\
, 049,95 & .38 \\
, 361.59 & .38 \\
, 219.76 & .42 \\
, 665.12 & .36\end{array}$ \\
\hline
\end{tabular}

Tabella V - Valori finali, nell'attuale sistema di Potsdam.

4. Basi di taratura per gravimetri. - Per l'opportunità di poter tarare i gravimetri a piccola scala nelle regioni stesse d'impiego, nonché di controllarne periodicamente in sito la costante di scala, è stata istituita una prima serie di basi di taratura convenientemente distri- 
primo contributo per una Rete cravimetrica fondamentale in italia

Tabella VI - Basi di taratura per gravimetri.

\begin{tabular}{|c|c|c|c|}
\hline Stazione principale & Estremi della base & $\mathbf{n}$ & $\Delta_{\mathrm{g}}(\mathrm{mgal})$ \\
\hline \multicolumn{4}{|c|}{ A. A piccola scala } \\
\hline \multirow[t]{2}{*}{ Catania } & $\begin{array}{l}\text { Osservattorio } \\
\text { Gravinal }\end{array}$ & 2 & $-83.23-0.05$ \\
\hline & $\begin{array}{l}\text { Osservatorio } \\
\text { Mascalucia }\end{array}$ & 5 & -99.66 \\
\hline Milano & $\begin{array}{l}\text { Politecnico } \\
\text { Pavia }\end{array}$ & 6 & $-46.38 \quad .02$ \\
\hline Padova & $\begin{array}{l}\text { Monselice } \\
\text { M.te Ricco }\end{array}$ & 6 & -72.78 \\
\hline Palermo & $\begin{array}{l}\text { Aereoporto } \\
\text { Monreale }\end{array}$ & 6 & -49.86 \\
\hline \multirow[t]{4}{*}{ Roma } & $\begin{array}{l}\text { I. N. G. } \\
\text { Frascati } \\
\text { Rocra di Papa inf. } \\
\text { Rocea di Papa Oss. }\end{array}$ & $\begin{array}{l}4 \\
4 \\
4\end{array}$ & $\begin{array}{ll}-71.88 & .06 \\
-70.65 & .01 \\
-28.24 & .02\end{array}$ \\
\hline & \multirow{2}{*}{\multicolumn{2}{|c|}{$\begin{array}{l}\text { Totale I. N. G. - Rocca di Papa: } \\
\text { Media } 6 \text { colleg. diretti (vite gr.): }\end{array}$}} & $-170.77 \div 0.06$ \\
\hline & & & $-170.69 \quad .14$ \\
\hline & \multicolumn{2}{|c|}{ Valore finale: } & $-170.74-0.05$ \\
\hline Trieste & $\begin{array}{l}\text { Oss. Geofisico } \\
\text { Opicina }\end{array}$ & 20 & -69.61 \\
\hline \multicolumn{4}{|c|}{ B. A grande scala. } \\
\hline Trento & $\begin{array}{l}\text { Zambana } \\
\text { Fai } \\
\text { Paganella }\end{array}$ & $\begin{array}{r}12 \\
8\end{array}$ & $\begin{array}{rr}-128.69 & 0.01 \\
-181.82 & .02\end{array}$ \\
\hline
\end{tabular}


buite (v. fig. 1). Gli elementi ad esse relativi sono riportati nella tabella VI con il numero $n$ dei collegamenti indipendenti e le differenze di gravità misurate.

Per i gravimetri a grande scala, il confronto avrebbe potuto essere effettuato su uno dei lati più sicuri dei poligoni della fig. 1: per es., Padova-Roma, o Roma-Palermo. Ma per una migliore precisione della base stessa, e per una maggiore comodità dei controlli, si è pensato ancbe di istituire una base su un forte salto in quota, da percorrersi in pochi minuti. E come tale si è scelta, per la facilità di accesso e per la massima differenza di quota nella zona, la funivia della Paganella. Con un salto verticale di $1600 \mathrm{~m}$ si è ottenuta così una differenza di gravità di $311 \mathrm{mgal}$, corrispondente all'incirca allo spostamento in latitudine da Milano a Napoli, e da Roma a Catania. E la precisione di questa base non è inferiore a quella risultante dalla differenza delle migliori stazioni fondamentali : tenendo conto dell'incertezza del $0,35 \%$ delle costanti di scala, essa si può assumere senz'altro precisa entro il $0,4 \%$.

5. Confronto con $i$ valori pendolari e gravimetrici precedenti. Pùo avere qualche interesse il confronto fra le differenze di qravità risultanti dalla presente ricerca e quelle osservate da altri autori. Espo. niamo i risultati di tali confronti conservando l'ordine precedentemente seguito.

A) Stazioni fondamentali e intermedie. - Per le stazioni fondamentali, il confronto per Padova, Milano e Roma è già stato effettuato in ( $\left.{ }^{2}\right)$; Trieste, Palermo e Catania sono stazioni nuove. Rimanơono quindi le seguenti differenze:

Roma I-Napoli I: $\Delta g$ Morelli $1951=-119,92 \mathrm{mgal}$;

$\Delta g$ precedenti : Ciscato 1908 (pendol.) $=-123 \quad$ " ;

Cassinis $1919($ ( ) $=-123 \quad$ " ;

Aquilina 1935 ( $) \quad$ ) $=-120$ " ;

Boaga 1951 (gravim.) $=-120.7 \%$ " .

'Tenendo conto dell'imprecisione dei metodi delle misure pendolari antiche, l'accordo è molto soddisfacente per le prime due; è addirittura ottimo per la terza; e denota una differenza dovuta probabilmente alla costante di scala per la quarta (v, anche $B$ ).

$$
\begin{array}{clll}
\text { Padova-Cagliari: } & \Delta g & \text { Morelli } 1951 & =-550.05 \mathrm{mgal}: \\
\Delta g \text { precedenti: } & \text { Boaga } 1937 \text { (pendol.) } & =-533 \quad \text { ") . }
\end{array}
$$

L'edificio dove il prof. Boaga ha fatto stazione a Cagliari è andato 
distrutto durante la guerra, per cui un confronto esatto non è possibile. La stazione 1951 è stata fatta su quello che doveva presumibilmente essere il pianoterra dell'edificio, fra le macerie.

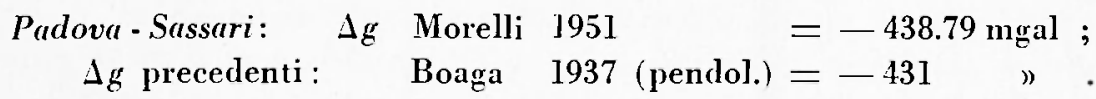

Fra le stazioni intermedie, Brescia, Verona e Trento coincidono con le stazioni effettuate con gravimetro $\left({ }^{3}\right)$, le cui differenze di gravità da Milano risultano dallo specchio che segue:

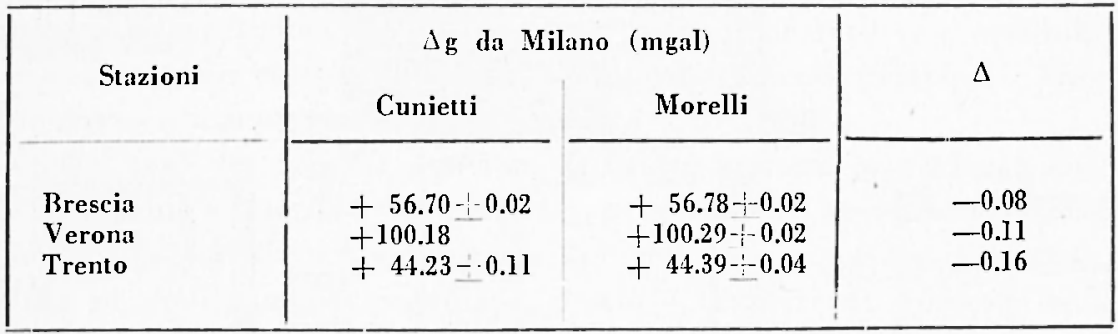

Tenendo conto che i collegamenti del Cunietti sono stati effettuato con un solo gravimetro (Western, W.48), non tarato, l'accordo è abbastanza soddisfacente.

B) Basi di taratura. - Delle basi di taratura, due hanno gli estremi in stazioni pendolari, e precisamente:

Milano-Pavia: $\quad \Delta g$ Morelli $1951=-46.38 \mathrm{mgal}$;

$\Delta g$ precedenti (3): Solaini e a. 1947 (pendol.) $=-49.7 \mathrm{mgal}$;

Cunietti 1949 (grav. W.11) $=-46.20$ ) .

La discrepanza pendolare ̀̀ un po' troppo forte per misure moderne, ed è probabilmente dovuta a impercettibili movimenti dei coltelli nelle loro sedi, cbe hanno alterato le misure pendolari del 1947 anche nelle altre stazioni. Per il gravimetro W.11 vale l'osservazione di sopra.

Roma-Rocca di Papa: $\Delta g$ Morelli ' $1951=-168.76 \mathrm{mgal}$;

$\Delta g$ precedenti ( ${ }^{4}$ ): Aquilina 1936 (pendolare) $=-169$ mgal ; Tribalto 1950 (grav. Western) $=-169.74$ » ;

Harding $1950($ ) Worden $)=-169.52$ » ;

Di Cesare 1950 ( ) Atlas) $=-169.62$ ” .

Ciò dimostra ancora una volta l'accuratezza e la precisione delle misure pendolari del prof. Aquilina; e sembrerebbe indicare un errore 
Monografie delle stazioni gravimetriche n. 1-12.
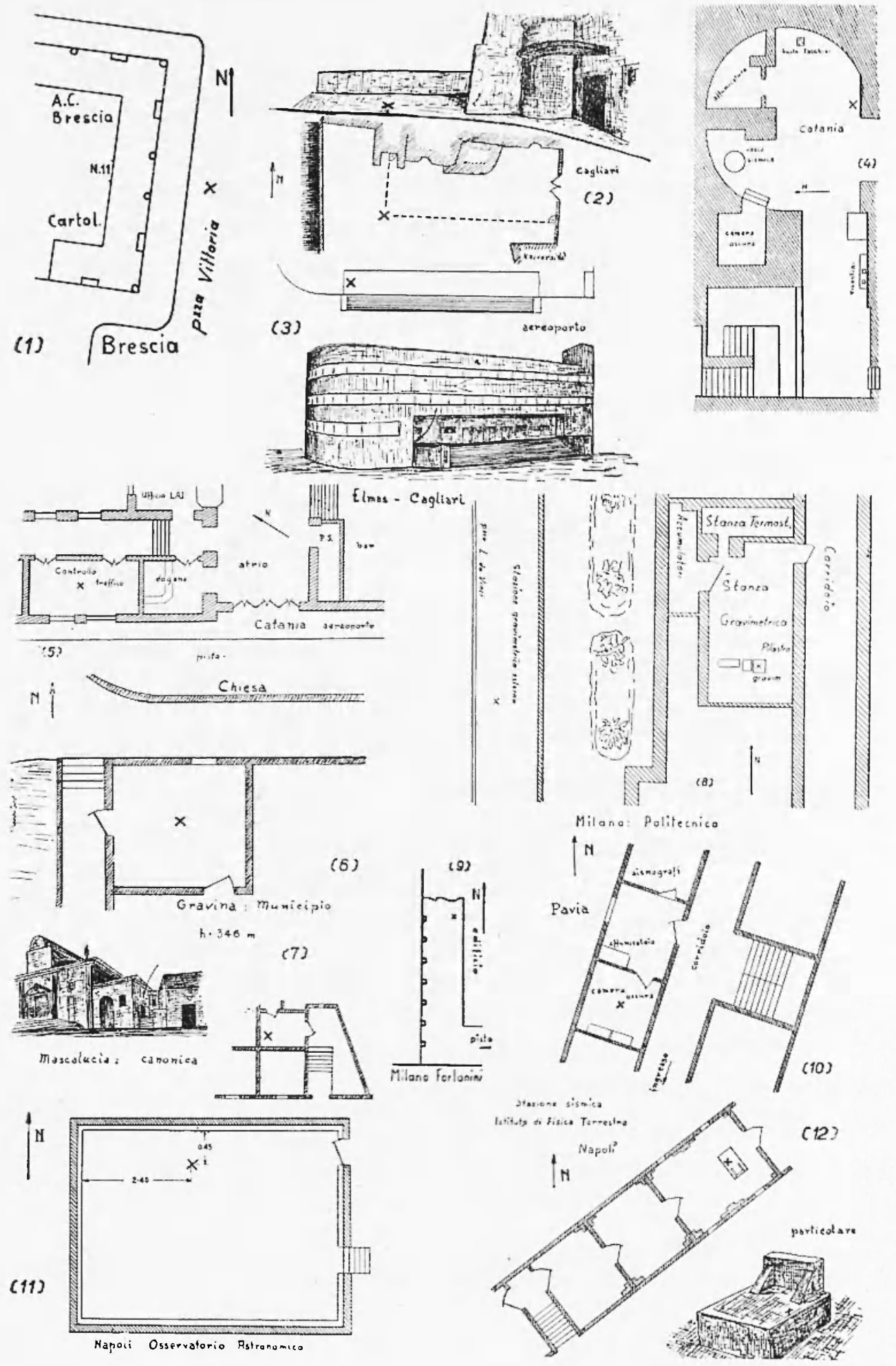
di taratura abbastanza cospicuo negli altri gravimetri (che però sono in buon accordo fra di loroj.

6. Stazione per $i$ collegamenti internazionali. - Come abbiamo visto nella nota precedente $\left({ }^{2}\right)$, e per $i$ motivi ivi specificati, i collegamenti gravimetrici internazionali prendono attualmente grande sviluppo. Essi possono venire effettuati sia con i pendoli, che con gravimetri convenientemente tarati; per i motivi già visti in ( $\left.{ }^{2}\right)$, la preferenza viene ormai data ai gravimetri; ma ai pendoli, adoperati con $i$ criteri di alta precisione moderni a ciò necessari e con un conveniente numero di ripetizioni dei collegamenti in condizioni il più possibile diverse, rimane sempre il compito dei collegamenti principali. Esamineremo ora separatamente i due casi.

Nel caso dei pendoli, conviene anzitutto mettere in evidenza che la precisione "interna» di una rete con gravimetri compensata come per esempio quella sopra illustrata, è certamente superiore alle possibilità dei collegamenti pendolari: perciò è indifferente collegare con l'estero uno qualunque dei punti fondamentali della predetta rete. Senonché le misure pendolari esigono disponibilità di laboratorio e condizioni ambientali che non tutte le stazioni possono possedere,

In Italia, adatte a tale scopo sarebbero: Padova, stazione gravimetrica nazionale e per lunga tradizione luogo adatto anche per la taratura dei pendoli stessi, modifiche strumentali, ecc.; e Milano, per l'attrezzatura disponibile all'Istituto di Geodesia del Politecnico.

Nel caso dei gravimetri, occorre anzitutto poter disporre di buoni aereoporti vicini; ed a questo scopo appunto sono state eseguite su quasi tutti gli aereoporti civili italiani le stazioni sussidiarie di cui sopra si è parlato. Data l'alta precisione consentita dai gravimetri, converrà poi inserire nei collegamenti internazionali le stazioni più sicure della rete fondamentale; e tali sono, nel sistema di Potsdam (v. fig. 1), Padova, Trieste, Roma e Milano.

Nella situazione attuale dei collegamenti aerei, la preferenza sembra andare a Roma specie per i collegamenti intercontinentali; anche Milano è però centro di importantissime linee aeree internazionali; e Trieste può servire egregiamente per il Centro-Europa. Palermo o Catania invece può essere comoda per i collegamenti con l'Africa.

Non sta naturalmente a noi di decidere in argomento; ma riteniamo non inutile questa breve rassegna, soprattutto per i Colleghi stranieri che ormai ablbastanza di frequente effettuano collegamenti gravimetrici con l'Italia. 
Monografie delle stazioni gravimetriche n. 13-26.
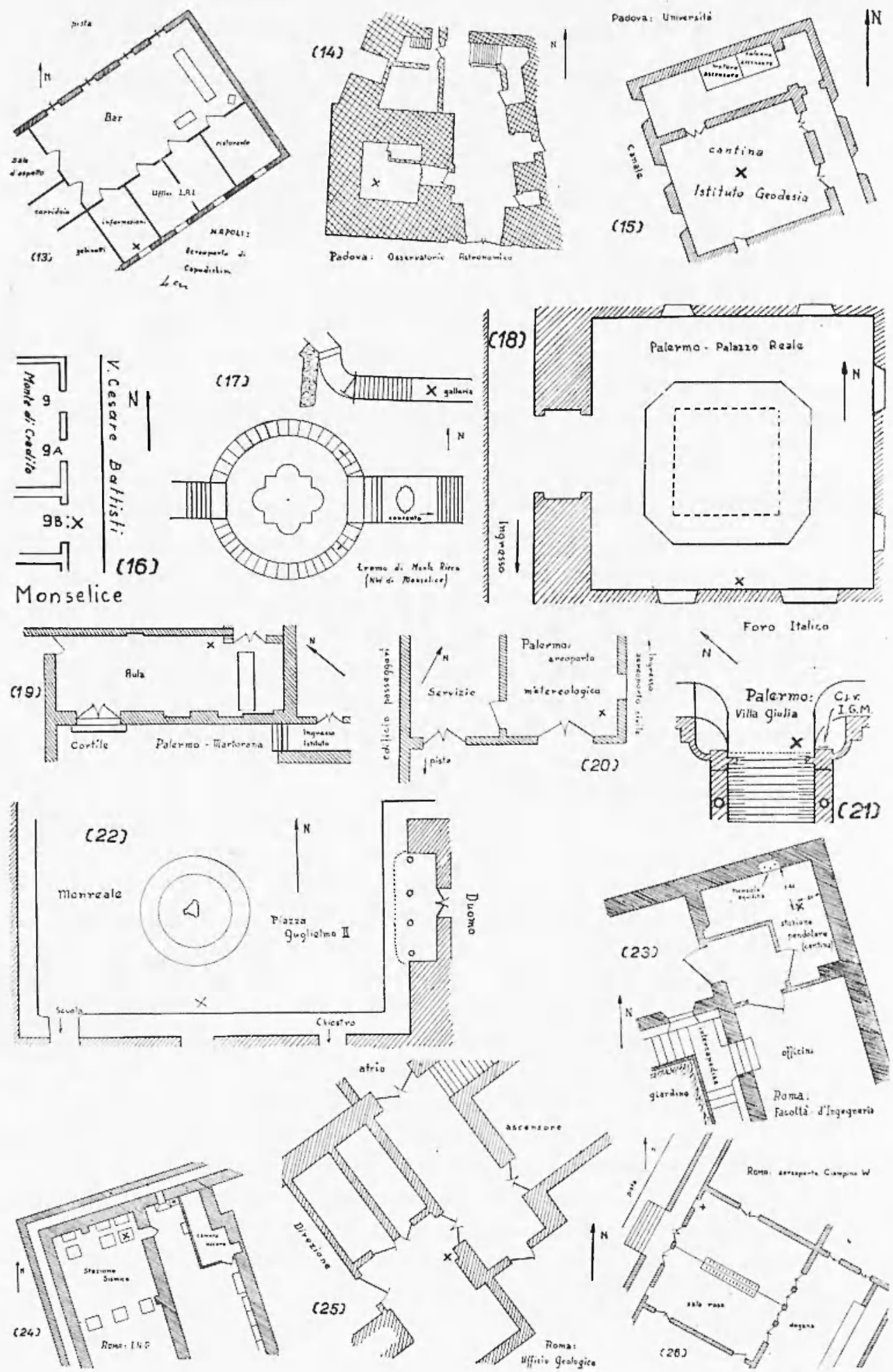
Conclusioni. - Da tutto quanto precede risulta chiaramente con quali vantaggi il gravimetro possa, in determinate condizioni esplicitamente specificate, sostituire il pendolo nei collegamenti gravimetrici. Il pendolo stesso non potrà però mai essere sostituito per la prima taratura dei gravimetri: e controlli successivi saranno sempre oltremodo desiderabili. In particolare per la rete italiana qui istituita una serie di misure pendolari molto accurate fra le stazioni fondamentali alle latitudini estreme sarà eseguita non appena sarà pronta l'attrezzatura strumentale necessaria.

La rete fondamentale qui iniziata presenta una sola lacuna veramente grave, nell'Italia del SE, rimasta scoperta non essendo stato possibile ottenere un aereo militare per queste misure $\left({ }^{*}\right)$ : è però auspicabile che essa venga eliminata non appena venisse ripreso il servizio aereo Roma-Bari, o con altri mezzi.

Tutte le rimanenti regioni d'Italia possono essere facilmente collegate, con grandissima precisione $( \pm 0.03 \mathrm{mgal}$, con una delle stazioni fondamentali qui istituite, ed essere quindi inserite nella rete nazionale.

Questa poi è già stata collegata nel suo complesso con Potsdam ( $\left.{ }^{2}\right)$, ed è auspicabile possa esserlo ancora nel futuro con altre stazioni nazionali di riferimento europee.

Istituto Naz. di Geofisica - Osserv. di Trieste - Dicembre 1951.

\section{A P P E N D I C E}

Successivamente alla consegna alle stampe di questo laroro, ma prima del licenziamento delle bozze, una ulteriore base di taratura renne richiesta dalla Commissione Geodetica Italiana nel tratto Bologna-Ferrara, dove si può ottenere su breve distanza un salto di gravità cospicuo sfruttando il forte gradiente gravimetrico derivante dall'anomalia dell'avampaese appenninico.

(*) Con l'aereo militare era prevista anche la chiusura completa di tutto il poligono esterno della rete, con l'esecuzione dei lati Cagliari-Palermo, Catania-Bari e Bari-Trieste. 
Monografie delle stazioni gravimetriche n. 26a-4l.
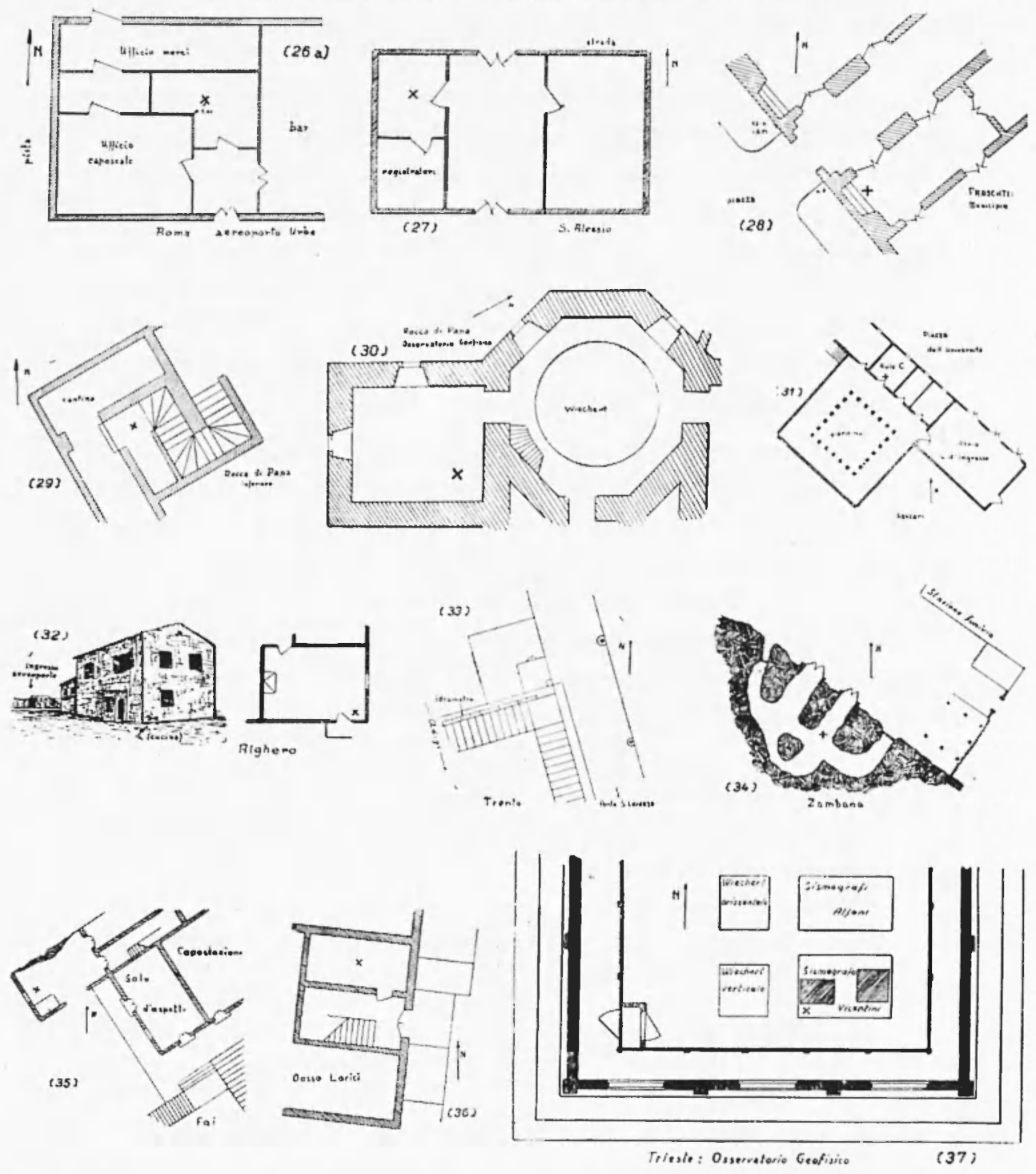

(38)
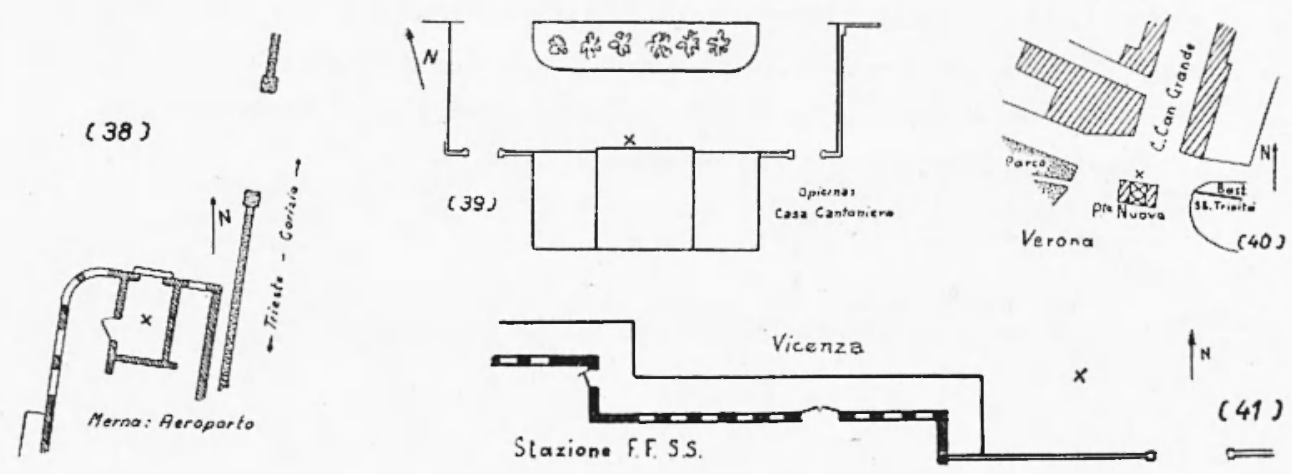
I caposaldi di tale base, stabiliti dal prof. Dore sulla S.S. n. 16, sono $i$ seguenti :

\begin{tabular}{|c|c|c|}
\hline Stazione & Descrizione & $\left|\begin{array}{c}\text { Vumero della } \\
\text { monografia }\end{array}\right|$ \\
\hline Bologna III & $\begin{array}{l}\text { Facoltà d'Ingegneria, in asse alla porta d'ingresso } \\
\text { dell'Istituto di Geodesia, sull'allineamento del } \\
\text { bordo del marciapiede }\end{array}$ & $(42)$ \\
\hline Argine & $\begin{array}{l}\text { a m } 3.0 \text { dalla porta n. } 135 \text { sulla via Nazionale, } \\
\text { localita Palazzacio (al } \mathrm{km} 109+\text { V) }\end{array}$ & (43) \\
\hline Altedo & $\begin{array}{l}\text { Sul bordo } W \text { della via Franchini, sull'allineamen- } \\
\text { to con la facciata } S \text { della casa } n .8\end{array}$ & (44) \\
\hline Malalbergo & $\begin{array}{l}\text { Entrando verso Est per il cancello al } \mathrm{km} 124+\mathrm{VII}, \\
\text { seguendo la stradicciola, appena passato il primo } \\
\text { ponte, a m } 10.5 \text { dallestremo E della spalletta S, } \\
\text { sul bordo merid. della strada }\end{array}$ & (45) \\
\hline Ferrara II & $\begin{array}{l}\text { Acquedotto: in asse all'ingresso isul fronte me- } \\
\text { rid.) a m } 10.6 \text { dal cancello, sull'allineamento col } \\
\text { bordo del marciapiede }\end{array}$ & (46) \\
\hline
\end{tabular}

Le medie delle differenze di gravità osservate con i due gravimetri sono risultate:

\begin{tabular}{|c|c|c|c|}
\hline Stazione & $\mathbf{n}$ & $\Delta_{\mathrm{g}} \quad(\mathrm{mgal})$ & EPotsdam \\
\hline $\begin{array}{l}\text { Bologna III } \\
\text { Argine } \\
\text { Altedo } \\
\text { Malalbergo } \\
\text { Ferrara III }\end{array}$ & $\begin{array}{r}12 \\
8 \\
12 \\
10\end{array}$ & $\begin{array}{rr}+50.32 & 0.01 \\
+33.24 & .01 \\
+30.27 & .01 \\
+47.23 & .01\end{array}$ & $\begin{array}{r}980,442.33 \\
, 492.65 \\
, 525.89 \\
, 556.16 \\
, 603,39\end{array}$ \\
\hline
\end{tabular}
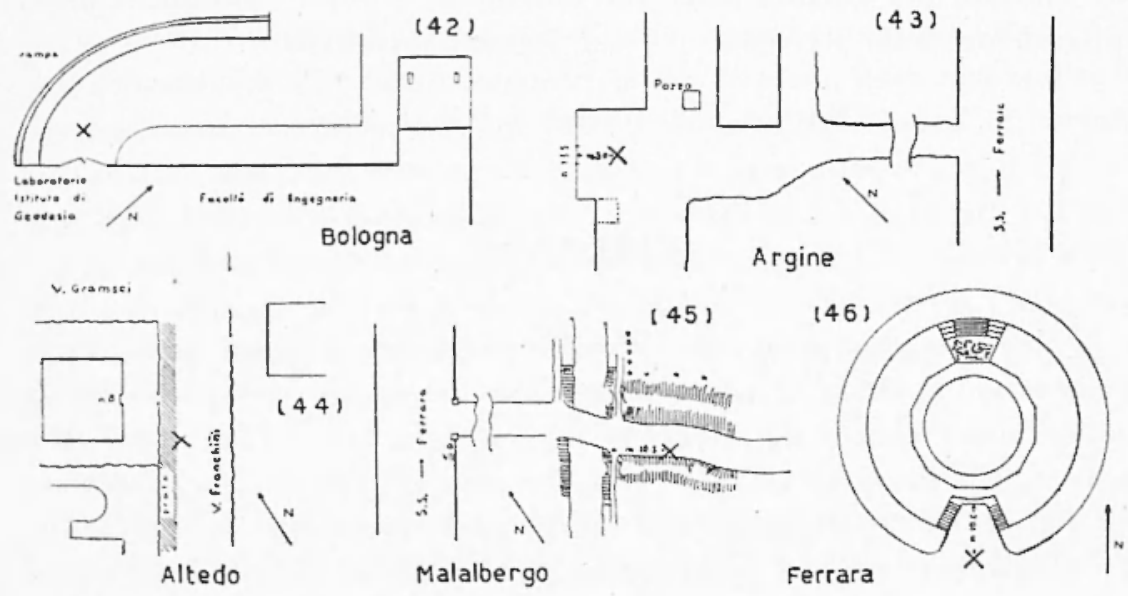
I valori di g nel sistema di Potsdam risultano dal collegamento del nuovo punto a Ferrara con la base di Padova, coi seguenti valori:

\begin{tabular}{|c|c|c|c|}
\hline Stazione & n & $\mathrm{Ag}$ (mgal) & El'otarlam \\
\hline $\begin{array}{l}\text { Ferrara II } \\
\text { Rovigo II } \\
\text { Monselice }\end{array}$ & $\begin{array}{l}4 \\
4\end{array}$ & $\begin{array}{rr}+ & 42.45-0.02 \\
+ & 31.63 \quad .02\end{array}$ & $\begin{array}{r}980,603.39 \\
, 645.84 \\
, 677.47\end{array}$ \\
\hline
\end{tabular}

Come controllo, si ha:

\begin{tabular}{|l|c|}
\hline \multicolumn{1}{|c|}{ Stazione } & Ig (mgal) \\
\hline Padova I & -216.22 \\
Bologna III & $\begin{array}{c}-11 \\
\text { Bologna II } \\
\text { Milano I } \\
\text { Padova I }\end{array}$ \\
\hline Errore di chiusura: & +9.56 .05 \\
\hline
\end{tabular}

La stazione di Bologna II è quella già occupata dal Cunietti (j) e dallo scrivente $\%$. nella stanza accanto a guella dei sismourafi al pianterreno dell'Istituto di Geodesia. Pure da precedenti collegamenti dello scrivente $\left({ }^{6}\right)$ risulta il valore della differenza Bologna II - Milano I sopra riportata.

La stazione Rovigo II qui istituita (Scuola di V ia Miani : davanti al cancello del cortile, sulla via Corridoni) ì stata collergata con la precedente dello scrivente $\left({ }^{6}\right)$, col seguente risultato:

Rovigo II = Rovigo I +0.31 mgal.

\section{RIASSUNTO}

Mediante due gravimetri $\pi$ orden previamente tarati con grande precisione $(0,35 \%)$ e convenientemente impiegati (trasporto aereo. collegamenti chiusi nel minor tempo possibile, ecc.), l'Istituto Nazionale di Geofisica ha istituito in Italia una prima rete fondamontale di stazioni di riferimento, distribuite opportunamente in latitudine. I collegamenti eseguiti costituiscono tutti poligoni chiusi, e gli errori 
di chiusura non superano mai $0.12 \mathrm{mgal}$. Come esempio della possibilità di compensazione, è stato applicato il metodo dei minimi quadrati al qualrilatero completo Roma-Napoli-Catania-Palermo: l'errore medio di un collegamento è risultato $0,04 \mathrm{mgal}$. In conseguenza dell'alta precisione della rete, una qualunque delle stazioni fondamentali può essere utilizzata nei collegamenti internazionali. Per i futuri collegamonti aerei nazionali e internazionali, sono state inoltre istituite in quasi tutti gli aereoporti civili stazioni sussidiarie, collegate con grande cura con quelle principali. Sono state infine eseguite, con la precisione del $0,4 \%$, sei basi di taratura e controllo per $i$ gravimetri a piccola scala (3 nell'Italia settentrionale, 1 nell'Italia centrale e 2 in Sicilia), una per $i$ gravimetri a grande scala (Paganella), ed una per $i$ gravimotri di entrambi i tipi (Bologna-Ferrara).

\section{SUMMARY}

By means of tuo Worden gravity-meters, previously accurately calibrated (0.35\%), and conveniently used (transportation by airplain, connections closed in the shortest possible time, etc.), the National Institute of Geophysics has established in Italy a first hasic net of reference stations, which are convenienly distributed in latitude. All connections form closed polygons, uhose closure errors are never higher than $0.12 \mathrm{mgal}$. In order to obtain an example of the compensation possibilities, the minimum squares method has been applied to the whole quadrilapter Rome-Naples-Catania-Palermo: the average error of a single connection results $\pm 0.04 \mathrm{mgal}$. Owing to the great accuracy of the net, all fundamental stations can be used for international connections. For future connections by airplain, both national and international, auxiliary stations have been also established at almost all civilian aierfields, connected to the principal ones. With the greatest care have also been established six calibration and testing bases for small dial gravity-meters $(3$ in Northern Italy, 1 in Central Italy and 2 in Sicilyi, and two for large dial gravity-meters on the Paganella and near Bologna.

\section{BIBLIOGRAFIA}

(1) MoneuLi C.: Studio del gravimetro Torden n. 50 e sua applicazione per un rilievo geofisico di dettaglio alle foci del Timavo. "Annali di Geofisica ", IV, 2, Roma 1951. 
(2) Monflut C.: Taratura di due gravimetri Worden e collegamenti europet. "Annali di Geofisica », IV, 4, Roma 1951.

(3) Cunietti M.: 51 misure di gravitì relativa eseguite in Italia settentrionale nel 1949. "Rivista Geomineraria ", X, 2, 37-58, Milano 1949.

(4) Bonga G. e Tribalto G.: Il gravimetro Western G. C. n. 49 del servizio geologico dItalia. "Boll. Servizio Geol. d'Italia », vol. LXXII, 1950 (I), nota X.

(5) Cunietri M.: Collegamento gravimetrico delle stazioni di Padova, Bologna e Milano per mezzo di un gravimetro Western $G \& A$. Rivista di Geofisica appl, XI, n. l, Milano 1950.

${ }^{6}{ }^{6}$ Monelu C.: Rilievo gravimetrico e riduzione isostatica nell'Italia nordorientale. Tecnica Italiana, Nuova Serie, VI, n. 3 e 4, Trieste 1951. 